Therapie des akuten Koronarsyndroms

\title{
Time is muscle
}

- Das akute Koronarsyndrom (ACS) ist ein lebensbedrohliches Krankheitsbild. Entscheidend für die Prognose ist, dass rasch die richtige Diagnose gestellt und die optimale Therapie eingeleitet wird.

Bei akut einsetzenden linksseitigen Thoraxschmerzen muss immer an ein akutes Koronarsyndrom gedacht werden. Diese Symptomatik erfordert eine umgehende Abklärung mittels EKG und Troponin-Bestimmung. "Idealerweise sollten solche Patienten sofort in einer Chest Pain Unit vorgestellt werden", sagte Prof. Evangelos Giannitsis, Heidelberg.

Zeigt sich im EKG ein ST-Hebungsinfarkt, sollte der Patient unverzüglich in ein Katheterlabor zur perkutanen Koronarintervention gebracht werden. Dabei darf keinesfalls, weder prästationär noch innerhalb der Klinik, unnötig Zeit vergeudet werden. „Wenn die Door-needle-Zeit innerhalb der Klinik von 90 auf 150 Minuten steigt, erhöht sich dadurch die Mortalität um das 5-Fache", so Giannitsis.

Entscheidend für die Prognose von $\mathrm{Pa}$ tienten mit einem ACS ist neben der Koronarintervention der frühzeitige Einsatz von Thrombozytenaggregationshemmern, wobei heute die duale Thrombozytenaggregationshemmung der Standard ist. Durch die zusätzliche Gabe von Clopidogrel zu ASS konnte die kardiale Ereignisrate inkl. Tod zusätzlich um $20 \%$ gesenkt werden. „Wird statt Clopidogrel Prasugrel gegeben, so kann die Prognose nochmals um $19 \%$ verbessert werden", so Giannitsis. Je früher die Thrombozytenaggregationshemmer zum Einsatz kämen, umso besser sei die Prognose. Deshalb sei es sinnvoll, mit dieser Therapie bereits prähospital zu beginnen.

Angesichts dieser überzeugenden Studienergebnisse, die im Rahmen der TRITON-TIMI-38-Studie erhoben wurden, wird Prasugrel (Efient $\left.{ }^{\oplus}\right)$ in den neuen Leitlinien für die Therapie des akuten Koronarsyndroms mit der Empfehlungsklasse IB propagiert. Im Rahmen dieser Studie konnte bei Patienten mit einem akuten Koronarsyndrom, die einer sofortigen Koronarintervention unterzogen wurden, der kombinierte Endpunkt aus kardiovaskulären Tod, Myokardinfarkt und Schlaganfall von $12,1 \%$ auf $9,9 \%$ und das Risiko einer StentThrombose von $2,4 \%$ auf $1,3 \%$ gesenkt werden. Nur bei Patienten mit hohem Blutungsrisiko, Z.n. Schlaganfall, schwerer Blutung oder Operation innerhalb des letzten Monats, bestehender oraler Antikoagulation bzw. Thrombozytopenie empfiehlt sich weiterhin Clopidogrel als Kombinationspartner von ASS.

- Dr. med. Peter Stiefelhagen

Quelle: Media Workshop „ACS-PCl: rewind

2012, fast forward 2012", Frankfurt/Main, März

2012 (Veranstalter: Daiichi Sankyo und Eli Lilly)

\section{Weniger schwitzen, besser leben \\ Orales Anticholinergikum bei Hyperhidrose}

- Mit einer Prävalenz von 16,3\% ist die Hyperhidrose eine häufige Krankheit. Die für Patienten äußerst belastende Symptomatik kann wirksam mit einem oralen Anticholinergikum behandelt werden, wie eine aktuelle Studie bestätigt.

Am häufigsten manifestiert sich die fokale Hyperhidrose im Bereich der Axilla, den Palmar- und Plantarflächen. Prof. Berthold Rzany, Berlin, machte darauf aufmerksam, dass bei den eingesetzten oralen Anticholinergika lediglich für Methantheliniumbromid (Vagantin ${ }^{\circledR}$ ) bislang eine kontrollierte Pilotstudie mit Hinweis auf Wirksamkeit bei axillärer Hyperhidrose vorlag (Hund $M$ et al. J Dtsch Dermatol Ges 2004; 2: 343-9). Nun stellte er die Ergebnisse einer neuen kontrollierten Studie vor, an der 339 Patienten teilnahmen (Müller $C$ et al. J Eur Acad Dermatol Venerol 2012; accepted). Die Studienteilnehmer mit axillärer bzw. axillärer und palmarer Hyperhi- drose zeigten bei Studienbeginn in der Gravimetrie (Bestimmung der Schweißmenge unter standardisierten Bedingungen) einen Wert von mindestens 50 mg pro fünf Minuten. Die Patienten erhielten verblindet entweder $3 \times$ täglich 50 mg Methantheliniumbromid oder Placebo.

Nach vier Wochen hatte in der Gravimetrie die mittlere axilläre Schweißmenge unter Verum gegenüber Studienbeginn um 69 mg/5 Min. abgenommen, in der Placebogruppe um $31 \mathrm{mg} / 5$ Min. Bei 77,3\% der Patienten wurde die Schweißproduktion unter Methantheliniumbromid um mindestens $40 \%$ vermindert.

Der Dermatology Life Quality Index (DLQI) verbesserte sich in der Verumgruppe von 16,4 auf 9,7, im Placeboarm von 17,0 auf 12,2 Punkte. Analog waren die Verbesserungen signifikant im Hyperhidrosis Disease Severity Score (HDSS) mit einer Abnahme von 3,2 auf 2,4 Punkte unter Methanthelini-

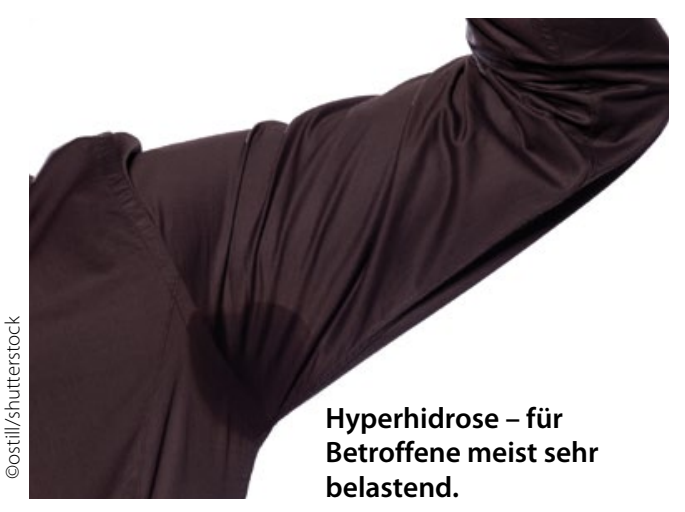

umbromid sowie von 3,2 auf 2,7 Punkte unter Placebo. Als häufigste Nebenwirkung gaben die Patienten Mundtrockenheit an.

Damit sei Methantheliniumbromid das für diese Indikation am besten dokumentierte orale Anticholinergikum auf dem deutschen Markt, fasste Rzany zusammen.

- Dr. Andreas Fischer

Quelle: Pressegespräch „Nutzen der systemischen Hyperhidrosetherapie - ein Update" 23. Fortbildungswoche für praktische Dermatologie und Venerologie, München, Juli 2012 (Veranstalter: Riemser) 